This is the final peer-reviewed accepted manuscript of:

Anna Rita Addessi, Laura Ferrari \& Felice Carugati (2015) The Flow Grid: A Technique for Observing and Measuring Emotional State in Children Interacting with a Flow Machine, Journal of New Music Research, 44:2, 129-144

The final published version is available online at: 10.1080/09298215.2014.991738

Rights / License:

The terms and conditions for the reuse of this version of the manuscript are specified in the publishing policy. For all terms of use and more information see the publisher's website. 


\section{The Flow Grid: A Technique for Observing and Measuring Emotional State in Children Interacting with a Flow Machine}

\begin{abstract}
The presence of flow state was measured and observed in children playing with the MIROR-Improvisation prototype, an interactive reflexive musical system (IRMS). IRMS have been defined as 'flow machines' on account of their ability to imitate the style of the human playing a keyboard. Twentyfour children (4 and 8 years old) took part in three sessions playing a keyboard on three consecutive days. In each session, all children played the keyboard with and without the MIROR-Impro, alone and with a friend. One group of children played the system with a set-up in which the reply is more similar to the child's input (set-up Same). The other group played the system with the set-up Very Different, in which the reply is less similar to the child's input. The results show that the flow state is higher when the children play with MIROR-Impro, with the set-up Same and in 8year-old children. These results would support the hypothesis that IRMS and reflexive interaction can generate an experience of well being and cre-ativity. The flow grid worked in an effective way and it was possible to identify some aspects of the system that could be improved.
\end{abstract}

Keywords: MIROR platform, flow machine, humanmachine interaction, observation methodology, emotion, childhood music education

\section{Introduction}

This article introduces an original grid measuring the flow experience (Csikszentmihalyi, 1996) of children interacting with MIROR-Improvisation (Pachet, Roy, \& Barbieri, 2011), one of the three software components of the MIROR platform, an innovative adaptive platform for childhood music and movement education based on the reflexive interaction paradigm implemented in the framework of the EU-ICT Project MIROR-Music Interaction Relying on Reflexion (Addessi \& Volpe, 2011). The interactive reflexive musical systems (in short IRMS) have been defined as 'flow machines' on account of their ability to imitate the style of a human playing a keyboard (Pachet, 2004). The flow theory has influenced studies on creativity in music (Addessi, Ferrari, Carlotti, \& Pachet, 2006; Byrne, McDonald, \& Carlton, 2003; Custodero, 2005; McDonald, Byrne, \& Carlton, 2006; Nijs, Moens, Lesaffre, \& Leman, 2012; O'Neill \& McPherson, 2002; Sheridan \& Byrne, 2002; Smolej Fritz \& Avsec, 2007) and is also considered one of the theories that best allows us to grasp the important aspects of human-machine inter-action (Finneran \& Zhang, 2005; Leman, Lesaffre, Nijs, \& Deweppe, 2010). The concept of flow machine integrates, in a very interesting technological hypothesis, the theories of creativity with the issue of human-machine interaction. It is therefore important to develop efficient tools to observe and quantify this particular kind of creative experience.

The study presented here has two objectives: on the one hand, it aims to verify the hypothesis that IRMS and reflexive interaction are able to enhance the flow emotional state in children and on the other, it aims to create and test a grid to analyse and measure the flow state of children interacting with a flow machine.

In this paper, we first introduce the MIROR platform and the reflexive interaction paradigm, as well as the theory of flow and studies on flow and music. We then describe the flow grid and the experimental design carried out with children interacting with the MIROR-Improvisation prototype. Finally, we present and discuss the results. 


\section{Background}

\subsection{The MIROR platform and reflexive interaction}

The MIROR Platform is an adaptive platform for childhood music and movement education made up of three components: MIROR-Improvisation (MIROR-Impro, hereinafter), MIROR-Composition and MIROR-Body Gesture. Each com-ponent aims to exploit the paradigm of reflexive interaction in the field of technology-enhanced learning. The reflexive interaction paradigm is based on the idea of letting users manipulate virtual copies of themselves, through specifically developed software called interactive reflexive musical sys-tems (IRMS) (Pachet, 2006). IRMS were born as music sys-tems. With the MIROR platform, the reflexive interaction paradigm is applied for the first time to motion capture tech-nology, giving rise to the MIROR-Body Gesture application (Addessi, 2013; Volpe, Varni, Addessi, \& Mazzarino, 2012).

\subsection{The interactive reflexive musical systems}

The first prototype of IRMS, the Continuator, was developed at the CSL-SONY in Paris, for adult musicians (Pachet, 2003). The idea was to develop a machine that gives users the per-ception of interacting with something similar to themselves. In this case, the machine does not exactly mimic the user's proposal, but her/his own musical style. The involved notion of style consists 'of the statistical distribution of notes, chords and musical elements in general as well as their ordering' (Pachet, 2004, p. 3), therefore placing itself within the body of studies that analyse style as a statistical phenomenon based on the recurrence of traits. But, unlike the best known systems developed in this field such as the one proposed by Cope (1996) - the Continuator is an agnostic tool, which learns new styles in real time and through interaction: 'The system we present here is an attempt to combine both worlds: real-time interactive musical instruments that are able to produce stylistically consistent music' (Pachet 2003, p. 333). The result of the sound interaction experience is that of a continuous back and forth process, during which the system mimics what has just been played by the musician who, in turn, repeats and varies what has been produced by the system, and so on.

Over the last two decades, other systems were developed to generate imitations of musical style: Assayag, Dubnov and Delerue (2004), and Triviño-Rodrigues and Morales-Bueno (2001) introduced new variants to the Markov model used by Cope (1996) with the idea to capture the local patterns found in the learnt corpus, using probabilistic schemes which generate the new sequences; Assayag and Dubnov (2004) applied a text sequence search algorithm (the Factor Oracle) to music for the learning and generation of pattern redundancy and variation. Maxwell, Eigenfeldt, Pasquier and Gonzalez Thomas (2012) proposed the MusiCOG, a novel system which models perceptual and cognitive processes in order to learn and generate music in a stylistically consistent way. Fober,
Letz and Orlarey (2007), instead, worked on the concept of mirroring systems with visual and aural feedback, in the field of instrumental music education (EU projects: IMUTUS and VEMUS). Recently, Gonzales Thomas, Pasquier, Eigenfeldt and Maxwell (2013) carried out an interesting study, comparing the output of three of these systems: the Continuator, the Factor Oracle system and the MusiCOG. The results suggest that the cognitive approach, i.e. the MusiCOG, would be the most successful at learning and generating melody in the style of the corpus used for the validation. However, unlike these systems that are able to learn and generate particular musical styles, the Continuator was conceived above all as a virtual musician, with which to undertake interesting musical dialogues in real time. Adult professional musicians have used the Continuator in the field of jazz and experimental music. Musicians and composers such as György Kurtág Jr., Bernard Lubat, Claude Barthelemy, have played extensively with the Continuator and performed in several festivals (Pachet, 2004). György Kurtág Jr. (Kurtág, Di Santo, Desainte-Catherine, \& Guillem, 2007) similarly drew inspiration from the Continua-tor, to implement a series of experiments on composition with children in the kindergarten.

More generally, the Continuator's aesthetic framework may be ascribed to an intertextual perspective, which has been circulating in the context of experimental and electronic music, particularly since the 1980s. This aesthetic framework is characterized by the fact that - in the original IRMS case - the machine and the musician deal with musical 'samples' taken from their own performances, in which the machine allows the musician to listen to and manipulate self-quotations in real time.

\subsection{Experiments with children}

The Continuator was originally invented for adult musicians. We now decided to use it with young children. The experiments with children and the Continuator (e.g. see Addessi \& Pachet, 2005, 2006; Ferrari \& Addessi, 2014; Pachet \& Addessi, 2004) immediately demonstrated the potential of these reflexive systems for the development of creative musical experiences also, and in a particular way, in young children. We observed that an innovative feature of the IRMS is the creation of a natural dialogue with the child. This dialogue is based on the mechanism of repetition and variation which is also a natural mechanism observed in infant-mother inter-actions (Bruner, 1983; Dissanayake, 2000; Gratier \& Apter-Danon, 2008; Imberty, 2005; Malloch, 2000; Papouš̌k, 1995; Stern, 2004; Trevarthen, 2000). The mechanism of repetition and variation is, in fact, at the heart of reflexive interaction: the system's repetition of the input given by the child allows the child to perceive the response of the system as a sort of sound image of her/ himself. As we have previously shown (Addessi \& Pachet, 2005), this is the moment when the child begins to show an absolute attraction towards this fascinating 'other'. The interesting thing is that it is not a mere imitation, but rather a repetition that is constantly varied. It is precisely the 
co-presence of something that is repeated along with something different that seems firstly to arouse the curiosity of the user, and subsequently to stimulate her/him to become more involved in this type of interaction. The remark 'It repeats but is different' expressed by a 5-year-old child after hearing the response of the Continuator for the first time, seems to sum up the attractive power of reflexive interaction.

During the course of our experiments it was possible to observe several interesting features that characterize the reflexive interaction: the attention of the child increases when the system imitates the child's input and decreases when the system's replies become more varied; the dialogue that emerges between the child and the machine is not predeter-mined by the machine, nor realized by the child alone, but is co-constructed by the child along with the machine; the co-regulation (Fogel, 2000) is based on a continuous repetition and variation mechanism between input and output data from the child and the system, where (a) the partners are able to imitate each other, (b) the child recognizes being imitated,(c) the repetition of something is always accompanied by the introduction of continuous variations. The interaction is thus based on turn-taking: the child plays, then stops, waiting for the response of the system and when it comes s/he listens to it carefully, perceives its reflexive qualities and responds by imitating and varying the system's response. This procedure is similar to what psychologists call the regular timing of turn and appears very close to that occurring in infant-adult interaction: in the exchange and vocal games of the child with the mother, the maternal voice acts as a sound mirror that reflects the vocal experience of the child and reinforces it.

\subsection{Towards a systematic perspective of the reflexive interaction paradigm}

Starting from the observation of children interacting with an IRMS, several theories have been put forward to explain the human behaviours engendered during the interaction with a reflexive system (Addessi, 2014). From a systematic perspec-tive, the theoretical framework of the reflexive interaction paradigm could include references ranging from the myth of Echo (Ovid) to the more recent semiological paradigmatic analysis (Meeùs, 1993; Nattiez, 1987; Ruwet, 1966) and the theory of similarity perception in listening to music (Deliège, 2001; Toiviainen, 2007). However, thanks to its ability to replicate musical behaviour and to evolve in an organic fash-ion with the user, the IRMS is able to translate several the-oretical concepts of learning development and the theory of creativity into a technological design. The ability to replicate the behaviour of others is to a certain extent grounded on the non-conscious processing known as the chameleon effect (Chartrand \& Bargh, 1999). Lakin and Chartrand (2003) sug-gest that the mere perception of another's behaviour automat-ically increases the likelihood of engaging in that behaviour oneself. Studies in neuroscience root these non-conscious mechanisms in the mirror neuron system (MNS), a network of neurons that becomes active during the execution and observation of actions (Rizzolatti, Fadiga, Gallese, \& Fogassi, 1998). The studies presented so far highlight the complexity of the processes set in place during a reflexive interaction such as that observed among children and the Continuator: imitation, imitation recognition, self-imitation, repetition and variation represent processes that develop in the first months of life and which structure the Self of the child and her/his interaction with the surrounding environment (Nadel \& Butterworth, 1999). Anzieu (1996) calls this kind of infant experience a musical wrapping of the Self, in which the Self is described as the first embryo of the personality felt as a unit, an individuality, and which expresses one of the most archaic forms of repetition: the echo.Another important aspect that we can draw from this literature is the importance of reflexive interaction as a dynamic process: the experience of repetition and variation is carried out within affective and emotional conditions, the amodal experience that Stern (2004) calls affective contours, which are the outcome of the child's experiences of interaction.

\subsection{Reflexive interaction and (music) pedagogy}

The basic hypothesis of the MIROR Project is that reflexive interaction (in short RI) enhances music learning and musical creativity in young children. We affirm that reflexive interac-tion could provide the grounds for a new model of (music) pedagogy in child-machine interaction and that the IRMS could represent a novel and original application of technology-enhanced learning. The pedagogical potential of RI lies in its ability to stimulate the subject to undertake a dialogue dur-ing which the repetitions and variations stimulate a cognitive conflict that the child solves during the course of the interac-tion, giving rise to learning by problem finding and problem solving. In our studies with children, we observed that the IRMS stimulated and reinforced exploratory conducts, during which the actions of the child were co-ordinated with the purpose of exploring the new partner and were characterized by the systematic introduction of new and different elements. Furthermore, it prompted inventive conducts, where the aim of the child's actions appeared to be to elaborate particular sounds and musical ideas and to undertake a dialogue with the system through the sounds. Both in the exploration and in the improvisation themselves, we saw very personalized styles in the children's approach to producing sounds, handling the instruments and other equipment, and in how they worked out plans of action to satisfy their own goals. The IRMS seem able to reinforce these individual styles, allowing them to develop and evolve. We observed turn-taking and regular timing of turns, the strategies of mirroring, modelling and scaffolding, and the starting up of affect attunement, intrinsic motivation, collaborative interaction and joint attention that characterize the 'teaching method' of the system. One of the most interesting aspects is that the invention is, in the end, not individual but collective: the child is playing along with the machine, in a pair, like two musicians improvising together. The way the children play also reveals their stylistic 
competence, not only as listeners, as previous studies found, but also as music-makers. We observed that RI increases the attention span, stimulates musical creativity, attentive listening, collaborative playing and ability in collaborative improvisation. IRMS also exploits the Vygotskian concept of zone of proximal development (ZPD) (Vygotsky, 1962). In this way, IRMS establishes an interaction between pairs, where the mirroring reflection creates a balance between challenges and skills, a basis upon which to create flow experiences and cre-ative processes. This characteristic would enable the MIROR Platform to enhance self-regulation, self-initiated activities, and the learner-centred approach.

\subsection{Analysing the reflexive interaction through the flow theory: the flow machine}

The ability of IRMS to imitate the style of a human playing the keyboard, and to maintain children's attention for long periods of time, has been interpreted through the theory of flow in-troduced by psychologist Mihaly Csikszentmihalyi (1996). In short, IRMS are defined as flow machines (Pachet 2006). Since this theoretical assumption had not yet found experimental and empirical support we decided to implement a tool able to empirically observe and quantify the optimal experience of children interacting with a flow machine.

According to Csikszentmihalyi (1990, 1996, 1997), and Csikszentmihalyi and Csikszentmihalyi (1988), the state of flow can be defined as the psychological state of maximum optimism and satisfaction that a person perceives during the course of an activity and it is closely related to the concept of creativity. The state of flow is defined as the optimal experience that results from the balance, perceived by the subject, between the challenges that s/he wants to achieve and the personal skills to achieve her/his goal. The flow is characterized by the presence of high levels of a series of variables, which are: focused attention, clear-cut feedback, clear goals, pleasure, control of situation, merged awareness, no worry of failure, disappearance of self-consciousness, change of the perception of time. According to Csikszentmihalyi's theory, in addition to the state of flow, other emotional states can be observed, defined as follows: arousal, control, boredom, anxiety, worry, relaxation and apathy. These emotional states are the result of different combinations of levels of the different variables.

Several studies applied the flow theory to the field of music education, performance and composition (Byrne et al., 2003; McDonald et al., 2006; Nijs et al., 2012; O'Neill \& McPherson, 2002; Sheridan \& Byrne, 2002; Smolej \& Avsec, 2007). Most of them are based on written interviews or ques-tionnaires, as in Csikszentmihalyi's research method. Instead, Custodero (2005) introduced observational indicators of flow state in young children's daily musical experience. This study was important because it defined in detail a series of observ-able indicators of flow to be grasped by the observer. Fur-thermore, it was developed in the particular field of childhood musical experience, thus providing teachers with a useful tool for flow observation in educational contexts.

In the field of embodied music cognition and humancomputer interaction Leman et al. (2010) indicate the theory of flow as one of the areas of expertise which should be explored to study human-machine interaction. They discuss various attempts to measure the flow experience, based on questionnaires (Jackson \& Eklund, 2004) and observational tools (Addessi \& Pachet, 2006; Custodero, 2005). Also several objective measurement methods based on behavioural and psychological aspects of flow are discussed, related to facial expression, posture response, eye movement, heart rate and skin conductance. More recently, Nijs et al. (2012) worked on the flow and its strong relationship with the concept of presence, as proposed by Riva, Waterworth and Waterworth (2004) in the field of virtual reality and ambient intelligence applications. The concept of presence is defined as the 'feeling of being in a world that exists outside the self but in which the self is situated' (Riva et al., 2004, p. 414) and it is used in combination with flow to study the impact of virtual reality and ambient intelligence in rehabilitative scenarios. Nijs et al.(2012) have emphasized the striking similarities between the constructs of flow and presence that occurred during the inter-action between clarinet students and the Music Paint Machine, an interactive computer system allowing a musician to make digital painting through the combination of sound and move-ment.

\subsection{Towards the grid measuring flow state}

While in Csikszentmihalyi's Experience Sampling Method (normally used by flow researchers), the subjects talk about their own sensations, in our study with young children the observers have to 'read' the reaction of children during their games with the keyboard. This methodological choice was due both to the greater effectiveness of the observation with regard to the age of the participants in our research (children aged two years and over), and to the fact that, in general, the observational method (unlike the interview) makes it possible to grasp several aspects of the subject's experience, which are not mediated by verbal language. In 2005, in their criti-cal review on the flow in computer-mediated environments, Finneran and Zhang (2005) had already pointed out the lack of studies capable of operationalizing and collecting quantitative data on the flow experience.

Our study is therefore the first to propose the use of the observational methodology, both to observe and to measure the flow experience in child-machine interaction. The flow grid introduced in this article takes its starting point from our previous study (Addessi et al., 2006), which proposed an observational grid measuring Csikszentmihalyi's variables and Custodero's indicators in 3 to 5-year-old children interacting with the Continuator. This study allowed us to observe that the interaction with this kind of system enhances optimal experience also in very young children. More precisely, it was observed that children were engaged in focused activity both 
when playing and listening (focused attention); they played with the system in a self-motivated way, without any external constraints; the Continuator produced clear feedback and the children's interaction was based on their analysis of the feedback produced by the machine; the children controlled the situation most of the time; the most striking result of the experiments (attention span, autotelic listening, Aha Effect) was related to the intrinsic motivation of the children. Excitement was clearly evident most of the time, in particular in the early phases of the sessions. We also noticed the presence of the flow indicators established by Custodero (2005), as follows: self-assignment, the activity is always initiated by the children (priority of the user); self-correction, during the interaction the children learn the implicit rules, assess their errors and correct them (for example in turn-taking); deliberate gesture, the children's movements are very focused and controlled, both during the listening and the playing; anticipation, the interaction based on turn-taking and repetition and variation allows the children to anticipate something of the system's reply, and to play on the basis of this anticipation; expansion, the children progressively modified the material, reaching a good ability in organizing the time; extension, the children always continue to work with the material after the system (the teacher in Custodero's indicator) has finished; awareness of adults and peers, both in the task alone and in pairs, we noticed that attempts to involve another person (and the system itself) physically or verbally are especially noteworthy.

In our previous study we made a systematic observation of flow using an observation grid, and found that the percentage when the flow state was present is higher in task B, with the Continuator (54\%) than in task A, without the Continuator (42\%) (for more details seeAddessi et al., 2006). Based on this experience we decided to create a more robust grid to observe and measure the flow. This step allowed us to strengthen the previous qualitative observations by adopting mixed meth-ods based on qualitative and quantitative analysis (Teddlie \& Tashakkori, 2009). The mixed methods, in fact, can simul-taneously address a range of confirmatory and exploratory questions with both the qualitative and the quantitative app-roaches. This approach also aimed to create, at the same time, a more precise and flexible tool that would allow us not only to observe the flow, but also to correlate the experience of the flow to other different variables, such as: the use of the system, the kind of system response, the different set-up of the system, children's age, if they play alone or with others, and so on. Finally, this procedure should allow us to identify in a more sophisticated way several problems that occurred in the child-machine interaction, highlighting usability and user requirements (Leman et al., 2010).

\section{Measuring flow}

The grid for observing and measuring the flow state of children playing with MIROR-Impro was created using the Observer software (@ Noldus). The basic idea of this grid was that the observer did not register the flow state, but rather considered the variables and their intensity. The presence of flow was instead measured by means of an automatic process of the Observer based on several constraints given to the software through the 'data profile' tool. The grid codes the following variables described by Csikszentmihalyi here defined as 'behaviours' (Figure 1): focused attention, clear-cut feedback, clear goals, control of situation, and pleasure.

The value, that is the level of intensity, of each behaviour was registered using the 'modifiers' tool (Figure 2), as follows: $1=$ low level of intensity: the child displays one or more features of the behaviour but in a not intense and piecemeal fashion, without continuing the behaviour on a continuous basis; 2 = medium level of intensity: the child displays one or more features of the behaviour with a medium intensity and frequency; 3 = high level of intensity: the child displays one or more features of the behaviour in a very clear, intense and persistent way over time. In some cases the behaviour may be very short but it may have a high intensity and directionality of the gesture and gaze.

The grid allows us to record over time the presence, the frequency, the duration, and the value of each behaviour. The Observer software calculated combinations of the different values of the behaviours, on the basis of several constraints

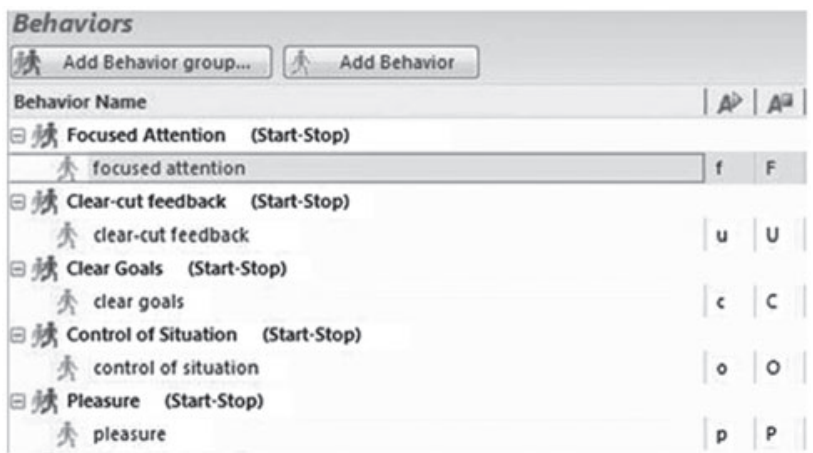

Fig. 1. Flow grid: The coding scheme showing the behaviours and, on the right columns, the start and stop keys used by the observers to register the behaviours (Observer XT 10.5-Noldus).

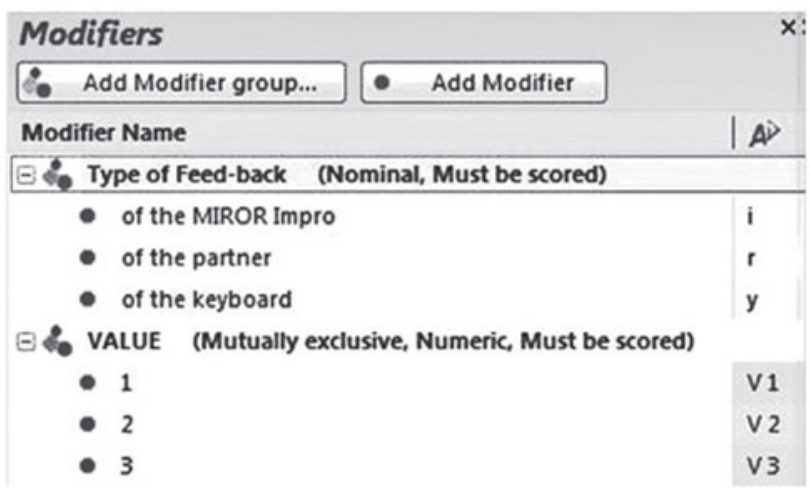

Fig. 2. Flow grid: the coding scheme showing the 'modifiers' and, on the right columns, the start and stop keys used by the observers to register the behaviours (Observer XT 10.5-Noldus). 
given by the researcher by means of the data profile tool. The data profile is essentially a series of consecutive 'nests', allowing us to identify the combined presence of pre-defined levels of the various behaviours. It was possible to extract the flow by means of a particular data profile: in accordance with Csikszentmihalyi (1996), when all behaviours showed high levels of intensity (modifier $=3$ ) the state of flow was indi-cated as present. Other combinations of levels of behaviours determined the state of arousal, control, anxiety, relaxation, worry, boredom and apathy.

\subsection{Definition of the behaviours}

The 'Instructions for the observers' included the practical actions required to register the behaviours using the software Observer, and the description of each behaviour, as follows.

\subsubsection{Focused attention}

Focused attention is an analytic behaviour of great intensity, and is present when the child focuses on one or more particular elements. The child is not distracted by the environment. Some examples of behaviour that characterize focused attention are: the child looks attentively at the keyboard and/or other elements of the equipment (loudspeakers, monitors, cables, etc.); the child observes, s/he is attentive and systematically explores some parts of the keyboard or other equipment; the child systematically explores some gestures of sound production; the child is focused on a particular musical idea, s/he plays a particular rhythmic pattern on the keyboard, listens carefully to the response of the system, and when the system stops, the child responds, in turn, continuing to repeat and/or elaborate the rhythmic patterns; the child listens attentively to her/his own production, to the system's responses, or to the partner's production.

\subsubsection{Clear-cut feedback}

Csikszentmihalyi defines clear-cut feedback as 'internalizing the field's criteria of judgement to the extent that individuals can give feedback to themselves, without having to wait to hear from experts' (1996, p. 114). In our case, the system con-tinuously produces a clear-cut feedback and the childsystem interaction coincides with the analysis that the child makes of the feedback received from the system. In our observations we will also determine how the child analyses and feels the feedback received from the partner (in the task in pairs) and from the keyboard when s/he is playing. Some examples of clear-cut feedback: the child becomes aware of the system's response and s/he reacts by smiling or saying something; the child changes her/his musical proposal according to the response received from the system; the child learns the rules of the system and learns to judge whether the response of the system will respect these rules; selfcorrection (Custodero, 2005).

\subsubsection{Clear goals}

Clear goals are present in situations where 'the creative process begins with the goal of solving a problem that is given to a person by someone else or is suggested by the state of the art in the domain (...). In flow we always know what has to be done'(Csikszentmihalyi, 1996, p. 113). During the interaction with the system, there are no set goals: children spontaneously create goals during the interaction. The goals are clear when the child's behaviours are intentional and not accidental. When the aims are clear, the children show the intention to find and to try spontaneous strategies, ways to explore and play the keyboard. These behaviours are acted out in a systematic (repeating the gesture or sequence of gestures) and precise way (trial and error). Examples of behaviour that characterize clear goals: the child clearly aims to explore the parts of the keyboard and the elements of the equipment; the child clearly aims to explore different gestures to produce sounds: presses the keys with one finger, with an open hand, with the elbow, arm, head, producing glissando, etc.; the child clearly aims to explore the sounds of the keyboard and develop a musical idea; the child clearly aims to teach the system particular musical patterns.

\subsubsection{Control of situation}

Control of situation is present, according to Csikszentmihalyi, when 'we are too involved to be concerned with failure, like a feeling of total control' (Csikszentmihalyi, 1996, p. 112). That is, the child constantly checks (monitors) her/his own actions during the interaction with the system. Examples of behaviour that characterize the control of situation: the child understands quickly that s/he can interrupt the system when s/he wants; self-assignment (Custodero, 2005), where activities (exploration, invention of a musical idea, etc.) are started by the child; deliberate gesture (Custodero, 2005), where movements are well controlled, both during the listening and the playing; the child explores and uses the equipment spontaneously, independently and with agility; the child understands and knows how to use/manage the rules of the interaction with the system (turn-taking, s/he stops when s/he wants, inventing new rules of interaction and playing, etc.); the child manages and organizes the interaction and the game with the partner.

\subsubsection{Pleasure}

Csikszentmihalyi writes that the 'flow is an innately positive experience, it is known to produce intense feelings of enjoyment' (Csikszentmihalyi \& Csikszentmihalyi, 1988, p. 35). In our encoding, the behaviour pleasure also includes the state of excitement. Examples of behaviour that characterize pleasure: the child smiles and/or laughs, s/he is calm; the child shows no displeasure; the child repeats an action that s/ he likes to do, for example, exploring a musical idea, playing and listening to the system, making a particular gesture, playing sounds that create fun for her/him, alone or together with the partner, etc.; 
the child produces exclamations of pleasure, for example, 'it answers me!' or 'it's fantastic!' When pleasure becomes more intense and visible, the states of excitement can be observed by an increase in the intensity of the movements, an increase in the intensity of the gesture on the keyboard, an increase in the voice volume, etc.

\section{Method}

An experimental protocol was created with the aim to observe whether the presence and the trend of the flow in children are affected by the reflexive interaction, the age of children, if the child plays alone or with a friend, and the exposure to the system.

In order to establish whether the reflexive interaction affects the flow state, the children were invited to play the keyboard both with and without the system. It was hypothesized that when the child plays with the system the flow state increases. Furthermore, two set-ups of the system were used, Same and Very Different, which defined how close the system's answer is to the original input by the user. The set-up Same is supposed to be more reflexive than the set-up Very Different, as the system's output melody is musically much closer to the user's input melody. One group of children used the set-up Same (group A) and another group the set-up Very Different (group B). It was assumed that when the child plays with the setup Same the flow state increases. In order to investigate the variable of age, two groups of subjects were organized: one group of 4-year-old children and one group of 8-year-old children. In order to investigate the variable alone or with a friend, each child was asked to perform two tasks alone and two tasks in a pair. In order to investigate the variable of exposure, three sessions were realized on three consecutive days.

\subsection{Participants and design}

Twenty-four children were involved in the experiment: $n=$ 12, 4-year-old children; $n=12,8$-year-old children. According to the design 2 (4-year-old, 8-year-old) $\times 2$ (set-up Same, set-up Very Different), each group of children was administrated four tasks (see below). Group A $(n=6$, 4-year-old children; $n=6,8$-year-old children) used the set-up Same; group B ( $n=6$, 4-year-old children; $n=6$, 8 -year-old children) used the set-up Very Different.

The children were from two different schools (one kindergarten and one primary school) and all of them participated on a voluntary basis. Care was taken not to diverge from their normal daily routines. By organizing the protocol in such a flexible manner, we could fit the experiment into the children's normal school routine in a natural way, allowing us to observe them under everyday conditions and encouraging them to express themselves in a relaxed and spontaneous fashion.

\subsection{Equipment}

The equipment consisted of: MIROR-Impro prototype v. 2.5; a KORG X50 music synthesizer; a TOSHIBA-Tecra notebook (Windows 7, 64 bits); two amplifiers M-AUDIO AV30; a USB cable for the connection between the synthesizer and the notebook; a SONY video camera (recording in HD); a tripod for the video camera.

The following different set-ups of the MIROR-Impro were used: Same and Very Different.

The other parameters were programmed as follows: phrase threshold $400 \mathrm{~ms}$; Max legato ratio 0.5; keeping in memory only the last 10 inputs played by the user; transposition tool 'true'; system memory cleaned at the beginning of each task.

\subsection{Procedure}

The experiment was carried out in a kindergarten and in a primary school in Bologna (Italy). Several preliminary meetings were held with the headmaster and the teachers, in order to introduce the MIROR project and a synthesis of the activities, and for the organizational and administrative agreements, including the ethical issues and risk assessment. A preliminary collective meeting was held with each group of children. The meetings lasted about 40-60 minutes and involved groups of approximately 12-20 children each. The aims of the meeting were to get to know the children, introduce the experimental staff to them and prepare them for the experimental activities. During the meeting games were played, also involving the keyboard and the MIROR-Impro.

Each child then took part in three sessions on three consecutive days. Video and audio recordings were made in the small library of the primary school and in the atelier of the kindergarten (see the setting in Figure 3). The keyboard was placed on a table in front of the children and the portable computer on a nearby table. A video camera was positioned in front of the children. One collaborator worked with the video camera, while another worked with the children and the computer. The sessions were individual (one child) or in pairs (two children). The operator gave the assignment to the child (if necessary s/he turned on the computer), and while the child was working, s/he stayed in the same room

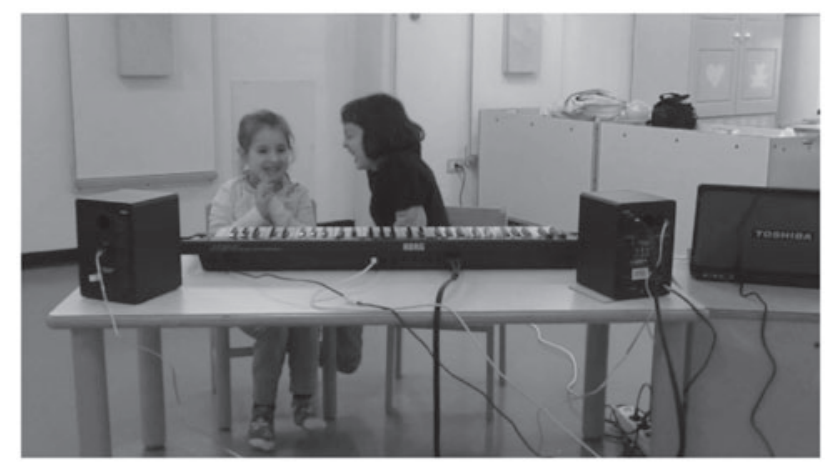

Fig. 3. Setting of the experimental protocol: two 4-year-old children, listening to the reply of the MIROR-Impro. 
and kept busy (reading, tidying). The children were asked to play the keyboard in four different ways: alone, alone with the MIROR-Impro, with another child, and finally with both another child and the MIROR-Impro. The operator asked the child to play the following 'musical games':

- Task 1. The child alone: 'Play the keyboard for as long as you like. When you are tired, call me';

- Task 2. The child alone with MIROR-Impro: 'Play the keyboard for as long as you like and it will answer you. When you are tired, call me';

- Task 3. The child with another child: 'Play the keyboard together for as long as you like. When you are tired, call me';

- Task 4. The child with another child and with the MIROR-Impro: 'Play the keyboard for as long as you like and it will answer you back. When you are tired, call me'.

For tasks 2 and 4 the operator activated the MIROR-Impro through the computer. With group A the operator activated the set-up Same, with group B the operator activated the setup Very Different. In session 1 the child was free to choose the first task; instead, the task order of sessions 2 and 3 was decided by the researchers (random order). All the sessions were video-recorded. The video camera was visible to the children. Before the beginning of the protocol the permissions signed by parents were collected. ${ }^{1}$

\subsection{Experimental hypothesis}

The basic hypothesis of the MIROR project is that the reflexive interaction and the mechanism of repetition and variation implemented by the IRMS could enhance creativity and learning processes. Consequently, the experimental hypothesis is that the flow increases when children play with the MIROR-Impro and with the set-up Same. Furthermore, we wish to investigate whether there is any correlation between the flow and the other independent variables: the age of children, the presence of the friend and the exposure to the system.

\subsection{Data analysis}

The flow grid, created with the software Observer, was used for the registration of the observation. Five independent observers registered their observation of the 'behaviours' and the 'modifiers'. Reliability tests among the observers were performed before starting the registration and during the registration. Any instances of disagreement were resolved by group

\footnotetext{
${ }^{1}$ Further data were collected which were used for other analyses besides flow. In particular: a week after session 3, each child was invited by her/his teacher to draw a picture of the experience; profiles of the children were completed by the teachers during the third week of the protocol; questionnaires for parents were distributed at the end of the protocol and collected by the teacher.
}

discussions and observations. The Observer software calculated combinations of the different levels of the behaviours: when all behaviours were recorded with high levels (modifier $=3$ ) the flow state was indicated as present. The statistical analysis ( $p, \mathrm{t}$-student, chi-square) was performed by SPSS.

\section{Results}

In general, the observed behaviours were similar to those observed in the pilot protocol (Addessi \& Pachet, 2005): the interactions between the machine and the children give rise to a series of creative behaviours, during which the children learn to interact with the system, explore the keyboard, the equipment, musical gestures and original musical ideas, work-ing them out together with the system and their schoolmates. In this article we intend to present the results related to the experience of flow.

The results indicate that the total duration of all tasks analysed is $27 \mathrm{~h}: 57 \mathrm{~mm}: 40 \mathrm{ss}$ and the duration of the intervals of flow scored for all subjects is $7 \mathrm{~h}: 08 \mathrm{~mm}: 05 \mathrm{ss}$. Thus, the flow state is present in $25.3 \%$ of the total duration of the tasks. If we consider that the optimal experience is not a state that appears suddenly but rather involves several elements developing in a dynamic way over time, and that the duration of the sessions also includes the beginning of interaction, as well as the time children need to become confident with the keyboard and manage the interaction with the friend and the setting, we can conclude that this percentage is very high.

Table 1 shows the duration (expressed in hours, minutes and seconds), the arithmetical average and the standard deviation of the total duration, the minimum and the maximum of the intervals of flow. It can be seen that the standard deviation of the minimum (3.15) and of the maximum (3.81) are lower compared with the standard deviation of the total duration (9.61). This result suggests that the duration of the single intervals of flow is approximately similar among the children. The higher value of the standard deviation of the total duration with respect to the standard deviation of the minimum and maximum could suggest that in some children the total number of the intervals of flow is higher than in other children. In conclusion, the duration of the intervals of flow is similar for all the children, while the total number of intervals varies from child to child. This result highlights that the evolution of the arrival and development of flow was experienced with a personal dynamic, in a space of time adequate for the cognitive and affective dynamic of each child and each pair of children. As the literature says, the temporal dynamic of experiences is fundamental to give affective meaning to human interactions (e.g. Imberty, 2005; Stern, 1995). This aspect, in our opinion, is very important not only for the study of the dynamic of arrival and maintenance of flow, but also because it would confirm the ability of the system to adapt to each user and group of users. 
Table 1. The flow within all sessions and all subjects.

\begin{tabular}{lccr}
\hline & D & $M$ & $S D$ \\
\hline Total duration & $07: 08: 05$ & $00: 17: 07$ & 9.61 \\
Minimum & $01: 25: 41$ & $00: 03: 26$ & 3.15 \\
Maximum & $03: 19: 39$ & $00: 08: 00$ & 3.81 \\
\hline
\end{tabular}

Note: This table shows the data concerning the total flow. The duration (D), the arithmetic average $(M)$ and the standard deviation $(S D)$ are indicated respectively for the total flow duration (Total duration), the duration of the shortest interval of flow (Minimum), and the duration of the longest interval of flow (Maximum).

\subsection{Flow state in all subjects for each task}

Figure 4 shows the percentage of flow registered in each task, that is, the total duration of flow compared with the total duration of each task. It can be seen that the percentage of flow is higher when the children play with the system, both with the set-up Same and Very Different, namely in task 2 and task 4 . The difference is significant when the child plays alone ( $p=0.004)$, whereas when the child plays with a friend, the difference is not significant ( $p=0.568)$. The latter result is particularly interesting because it is congruent with the theory of flow, which describes the flow as a subjective state. However, it should be noted that when the child plays in a pair with a friend (task 3 and task 4), the flow increases when the system is involved (task 4). This result confirms the result of the pilot protocol (Addessi et al., 2006), and reinforces the hypothesis that the IRMS can generate an optimal experience in the users, not only when they play alone, but also when the interaction is shared with others. From a socioconstructivist perspective of music education, this result is very important because it shows that this system is able to increase and reinforce the interaction among children and their sharing experience, enhancing socialization and collaborative playing.

In conclusion, the overall result for all subjects supports the experimental hypothesis that the flow state increases when the children play with the MIROR-Impro prototype, both when playing alone or in pairs.

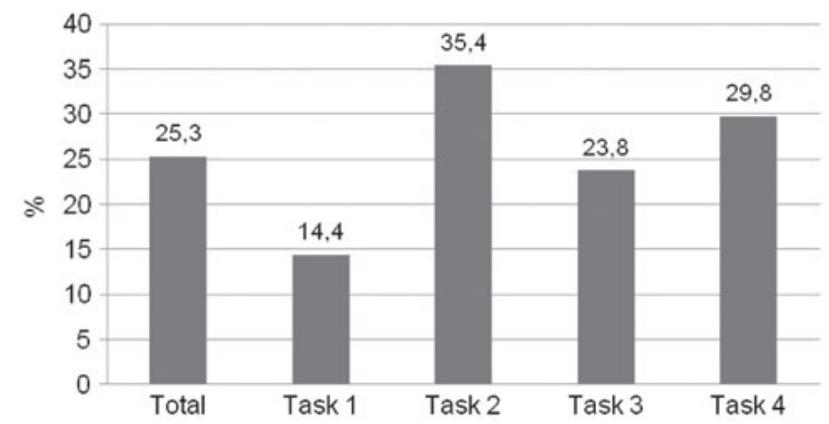

Fig. 4. Percentage of flow registered in total and in each task for all subjects.

Note: (subjects*3 sessions) T1*T2: $t=-3.012, d f=64$, $p=0.004$; T3*T4: $t=-0.574, d f=63, p=0.568$.

\subsection{Do the Same and Very Different set-ups affect the flow state?}

The results introduced in the previous sections were calculated on the total number of subjects, without distinction between group A, which used the more reflexive set-up (Same) and group B, which used the less reflexive set-up (Very Different). In this section we will present the results related to the difference between the two groups and therefore the influence of reflexive reply on the children's flow experience.

Figure 5 shows the percentage of flow in each task for group A (black column) and group B (grey column). The flow percentage is higher for group A, in all tasks, with or without the system. The difference between group A and group $\mathrm{B}$ is significant $(p=0.004)$. In particular, the difference is significant in tasks 2 and 4 (T2: $p=0.000$; T4: $p=0.001$ ), that is when the children used the system. Also in this case, we can observe that the flow is higher in tasks 2 and 4 (with the system), than in tasks 1 and 3 (without the system), and that the difference is significant, both in group $\mathrm{A}(p=0.035)$ and $\mathrm{B}(p=0.013)$. This result, and in particular the result related to tasks 2 and 4, would support the hypothesis that the flow state increases when the system's reply is more reflexive and similar to the input played by the children. Therefore, it

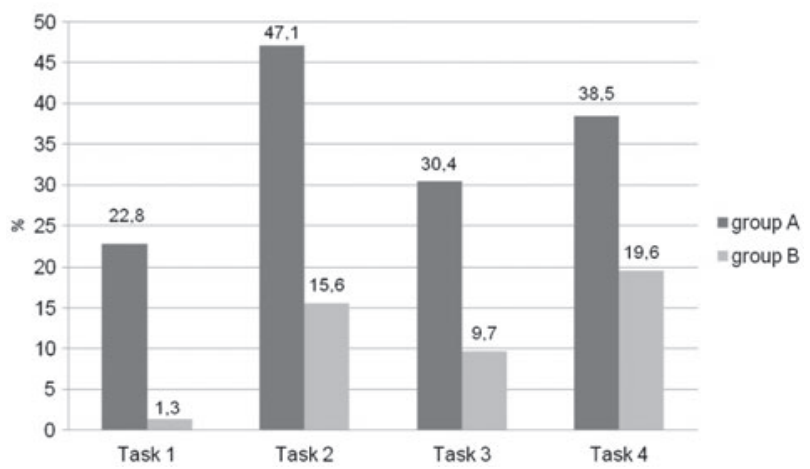

Fig. 5. Percentage of flow with group A and group B in each task. In the tasks with the system (T2 and T4) group A used the set-up Same and group B used the set-up Very Different.

Note: group A*group B: $t=8.151, d f=3, p=0.004$; T2 group A*group B: $\chi^{2}=21.685, d f=1, p=0.000 ;$ T4 group A*group $\mathrm{B}: \chi^{2}=10.214, d f=1, p=0.001$; group $\mathrm{A}(\mathrm{T} 2+\mathrm{T} 4) *(\mathrm{~T} 1+\mathrm{T} 3)$ : $\chi^{2}=4,434, d f=1, p=0.035$, group B $(\mathrm{T} 2+\mathrm{T} 4) *(\mathrm{~T} 1+\mathrm{T} 3)$ : $\chi^{2}=6.203, d f=1, p=0.013$; group A T1*T2: $\chi^{2}=11.159$, $d f=1, p=0.000$. 
is possible to say that the results support the main hypothesis of our study, that reflexive interaction and the interaction with IRMS enhance the flow state.

If we consider the variables 'alone' (tasks 1 and 2) and 'in a pair' (tasks 3 and 4), we can observe a difference between group $\mathrm{A}$ and $\mathrm{B}$. In fact, the result for group A confirms the previous data that the flow is higher when the child plays alone. It is also interesting to note that in group A the flow state increases not only when the system is used (tasks 2 and 4), but also during the tasks without the system (tasks 1 and 3). It could be argued that the use of the more reflexive set-up generally increases the children's attraction toward the keyboard and the music, enhancing their desire to play and their intrinsic motivation.

We compared the results of the pilot protocol (Addessi et al., 2006) with the results for group A, which use the MIRORImpro with the set-up Same, that is the set-up most similar to that of the Continuator system used in our previous study. We observed that in the pilot protocol the flow percentages are $42 \%$ without the system and $54 \%$ with the system. With the MIROR-Impro the flow percentages are $22.8 \%$ without the system and $47.1 \%$ with the system. Both experiments show a higher flow percentage in the task with the system and therefore confirm the main hypothesis of our studies. However, we can also observe some differences. For example, the flow is generally higher in the pilot protocol, both without and with the system, so it would appear that the Continuator was able to generate more flow than the MIROR-Impro. However, we can also see that with the MIROR-Impro the difference between task 1 and task 2 is high and significant ( $p=0.000$ ), while in the pilot protocol this difference is very low. It seems therefore that the MIROR-Impro affects the children's playing more than the Continuator did. These differences between the two experiments could be also due to the age of the children that participated (in the pilot protocol the age was 3, 4 and 5 years), to some differences in the settings, and finally to the grid itself, which was more efficient in the second experiment, as we will discuss later.

\subsection{Does the age of children affect the flow state?}

Figure 6 shows the percentage of flow in each task with the 4 (black column) and 8 (grey column) year-old children. The percentage of flow is clearly higher in the 8-year-old children. The difference between the 4 and 8 year-olds is significant ( $p=0.001$ ). These results support the hypothesis that during the interaction with the MIROR-Impro, the age could affect the presence of flow. Furthermore, we observe that when the child plays alone, the flow increases while playing with the system, in both 4 and 8 year-olds (4-year-old: $p=0.003$; 8 -year-old: $p=0.006$ ). In the task in pair ( 3 and 4$)$, the flow increases just a little in 8-year-olds, while it remains almost the same in 4-year-olds. In general, therefore, we can see that also the results on age confirm that the system increases the flow as a personal experience, but at the same time also enhances the flow in social experience and collaborative playing.

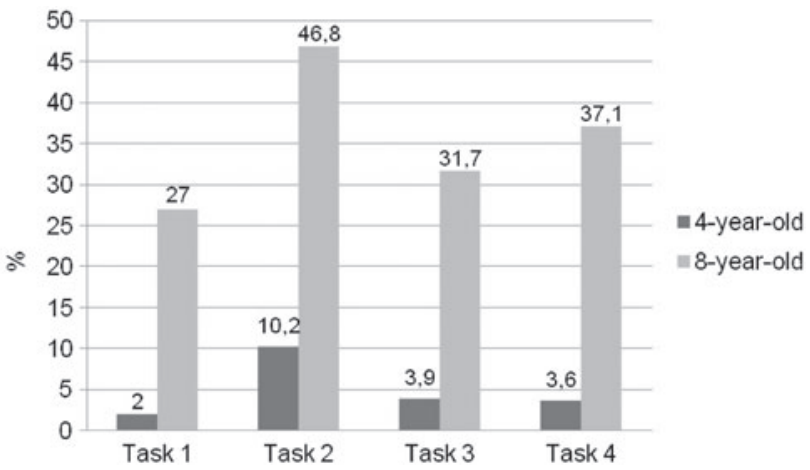

Fig. 6. Percentage of flow in each task with 4 and 8 -year-old children. Note: 4-year-old*8-year-old: $t=-11.676, d f=3, p=0.001$; age $4 \mathrm{~T} 1 * \mathrm{~T} 2: \chi^{2}=4.564, d f=1, p=0.033$; age $4 \mathrm{~T} 3 * \mathrm{~T} 4$ : $\chi^{2}=0.082, d f=1, p=0.775$; age $8 \mathrm{~T} 1 * \mathrm{~T} 2: \chi^{2}=7.644$, $d f=1, p=0.006$; age 8 T $3 * \mathrm{~T} 4: \chi^{2}=0.430, d f=1, p=0.512$

\subsection{Does the exposure to the system affect the flow state?}

Table 2 shows the percentage of flow for each session, in total and in each task. The flow remains almost constant between sessions $1(23.1 \%)$ and $2(22.9 \%)$, and increases in session $3(31.6 \%)$. We can therefore observe a positive trend between the first and the last session. The differences between the sessions are not significant (session $1 *$ session 2, $p=0.521 ; \operatorname{session} 2 * \operatorname{session} 3, p=0.285$ : session $1 *$ session $3, p=0.154)$.

Figure 7 shows the percentage of flow for each task and in each session. We have already pointed out that tasks 2 and 4 show the highest flow percentage. In this section we aim to show the evolution of flow over the three sessions in each task. It can be seen that the percentage of flow in task 1 (the child alone without the system) and in task 2 (the child alone with the system), increases over the three sessions; in task 3 (with a friend without the system) it decreases in the second session and increases in the third session, whereas in task 4 (with a friend with the system) the flow decreases in both the second and third sessions. It is therefore possible to observe that the flow state increases over the sessions when the child plays alone, while it is discontinuous when the child plays with a friend. This is congruent with the theory of flow that describes the flow as a subjective state, as we also noted in the results about the set-up, and with the previous experimental result from the pilot study.

Finally, it is possible to observe that the flow presence is more constant when the children use the system (tasks 2 and 4). The same trend was observed also in the pilot protocol (Addessi et al., 2006). These results not only confirm the hypothesis that the reflexive interaction increases the flow experience, but also that it maintains this experience constant over time.

\subsection{The other emotional states}

Besides the flow, different data profiles have been created to calculate the other emotional states: control, arousal, anxiety, 


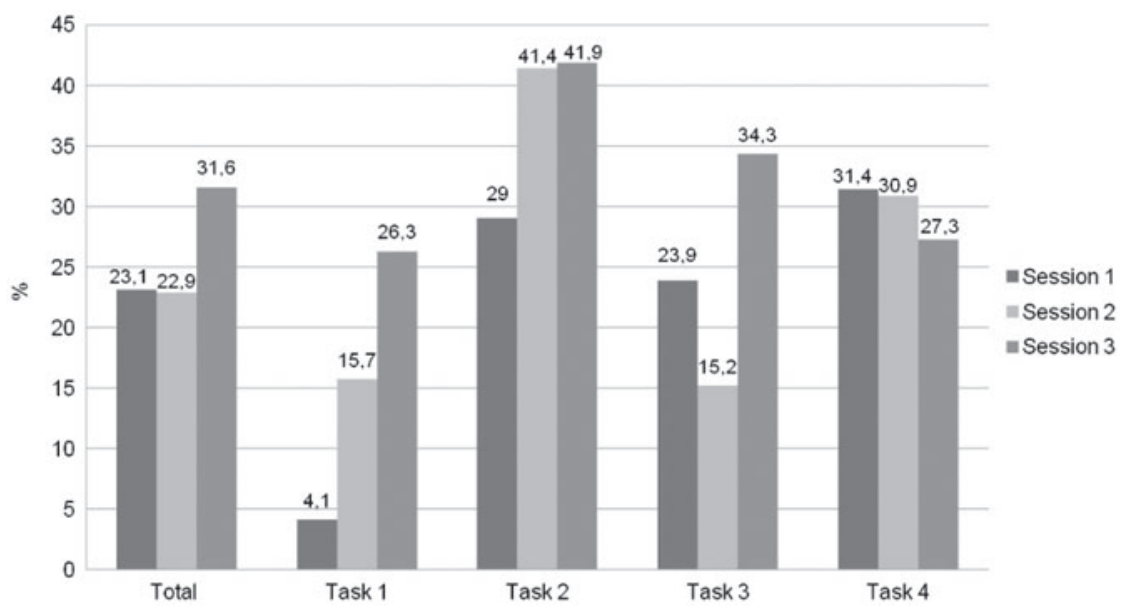

Fig. 7. Percentage of flow for each task in sessions 1,2 and 3.

Note: session $1 *$ session $2: t=-0.725, d f=3, p=0.521$; session $2 * \operatorname{session} 3: t=-1.299, d f=3, p=0.285$; session 1 *session 3 : $t=-1.899, d f=3, p=0.154$.

Table 2. Percentage of flow for each session, in total and in each task.

\begin{tabular}{|c|c|c|c|c|c|}
\hline & Total & $\mathrm{T} 1$ & $\mathrm{~T} 2$ & $\mathrm{~T} 3$ & $\mathrm{~T} 4$ \\
\hline Session 1 & 23.1 & 4.1 & 29 & 23.9 & 31.4 \\
\hline Session 2 & 22.9 & 15.7 & 41.4 & 15.2 & 30.9 \\
\hline Session 3 & 31.6 & 26.3 & 41.9 & 34.3 & 27.3 \\
\hline
\end{tabular}

Note: Session 1 *session 2:t $=-0.725, d f=3, p=0.521$; session $2 *$ session $3: t=-1.299, d f=3, p=0.285$; session $1 *$ session 3 : $t=-1.899, d f=3, p=0.154$.

Table 3. Combinations of the level of intensity of the five behaviours and the emotional states.

\begin{tabular}{|c|c|c|c|c|c|}
\hline & $\begin{array}{c}\text { Focused } \\
\text { Attention }\end{array}$ & $\begin{array}{l}\text { Clear-cut } \\
\text { feedback }\end{array}$ & Clear goals & $\begin{array}{c}\text { Control of } \\
\text { situation }\end{array}$ & Pleasure \\
\hline Flow & 3 & 3 & 3 & 3 & 3 \\
\hline Arousal & 2 & 2 & 2 & 1 & 3 \\
\hline Control & 2 & 2 & 2 & 3 & 2 \\
\hline Relaxation & 1 & 2 & 1 & 2 & 2 \\
\hline Anxiety & 3 & 1 & 3 & 1 & 1 \\
\hline Worry & 2 & 1 & 1 & 1 & 1 \\
\hline Boredom & 1 & 1 & 2 & 2 & 1 \\
\hline Apathy & 1 & 1 & 1 & 1 & 1 \\
\hline
\end{tabular}

Note: The values indicate the level of intensity of each behaviour: $3=$ high, $2=$ average, $1=$ low.

relaxation, boredom and apathy. In agreement with Csikszentmihalyi (1997), we created the combinations of the level of intensity of each behaviour for every emotional state, as shown in Table 3.

Figure 8 shows the percentage of flow, control, arousal, anxiety, relaxation, boredom and apathy scored in all tasks for all subjects, that is, the total duration of each emotional state compared with the total duration of all tasks analysed. It can be seen that flow $(25.3 \%)$ and control $(7.4 \%)$ have the highest percentage in all tasks. If we consider that control, like arousal, is a state that preludes to the achievement of flow, we can conclude that the presence of positive emotional states is quite high. In fact, Csikszentmihalyi (1997) describes control as a positive state for the individual, who feels satisfied and competent. Furthermore, the percentages of the presence of arousal, relaxation and boredom are similarly low (less than $1 \%)$. Episodes of apathy were scored $(1.5 \%)$. No episode of anxiety was scored (0\%).

\subsection{The behaviours: focused attention, clear goals, clear- cut feedback, control of situation and pleasure}

Table 4 and Figure 9 show the percentage and the trend of each of the five behaviours for all subjects, all tasks and all values. It can be seen that all behaviours reach a high level or presence. 


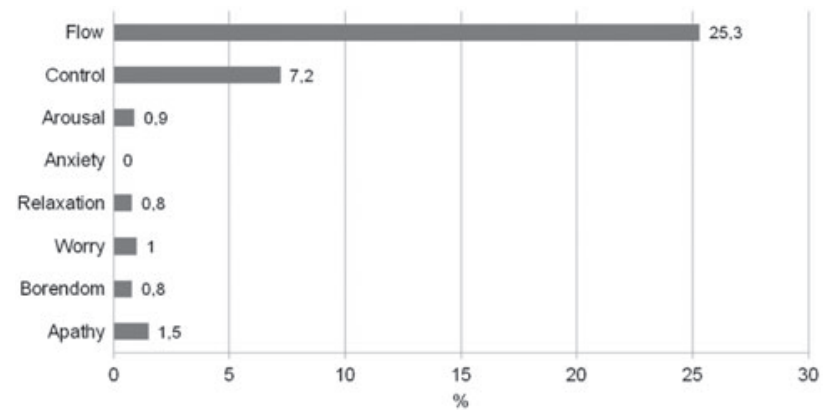

Fig. 8. The emotional states: Percentage of flow, control, arousal, boredom, relaxation, anxiety and apathy, in all sessions and all subjects.

Pleasure shows the highest percentage, followed by control of situation, focused attention, clear goals and clear-cut feedback. All behaviours, excluding pleasure, show the highest values in tasks 1 and 2 , that is when the child plays alone, with and without the system. Only pleasure shows the highest value in tasks 3 and 4, that is when the child plays with a friend, with and without the system. We can argue that sharing the playing with a friend enhanced the pleasure of the experience, while personal involvement seems to be required for implementing clear-cut feedback, focused attention, clear goals and control of situation. The percentages of all behaviours increase in task 2 , decrease in task 3 and increase in task 4 . All behaviours are higher in task 2, except pleasure, which is higher in task 4. The lowest presence of pleasure is in task 1, when the child plays the keyboard without a friend, without the system. These results are in line with flow theory, which considers happiness (pleasure) more a consequence of flow state than a typical variable, and also confirms that the flow is mostly experienced as a personal emotional state.

\section{Discussion}

As far as the reflexive interaction paradigm is concerned, the results suggest that the MIROR-Impro system and reflexive interaction can enhance flow in children, establishing the conditions for creativity processes. The results show that the flow emotional state increases not only when children play with the MIROR-Impro, but also when they play with the set-up Same, that is the more reflexive set-up used in the experiment. These results support the hypothesis that reflexive interaction creates a state of balance between challenges and skills, as described by Csikszentmihalyi (1996), which is the result of the continuous co-regulation started up between child/ren and IRMS. These results would also support, in terms of quantita-tive data, a wide range of qualitative observations related to the mechanism of mirroring, repetition and variation, imitation, turn-taking, and regular timing of turn, which characterize reflexive interaction, showing that they are able to create flow experience, well-being and creativity processes. From a peda-gogic point of view, this aspect is of utmost importance since it stimulates learning and creativity, as well as encouraging an interest in musical instruments (Burnard, 2006; Delalande, 1993; O’Neill \& McPherson, 2002). These results confirm the educational potential of the IRMS and of reflexive inter-action in the field of technologyenhanced learning. In this framework, the MIROR project aims to create a platform for children's music and dance education and creativity, by extending the reflexive paradigm into other domains, like those of movement and the visual arts. The exploitation of this paradigm in the field of technology-enhanced learning and more in general in the field of children's creativity is desirable, and new applications developing reflexive interaction could be added to the MIROR platform.

The fact that the presence of flow is more evident when children play alone with the system confirms the results of the pilot protocol (Addessi et al., 2005) and seems to be consistent with the theory of flow, which deals with personal creativity and subjective experience. However, this result does not mean that the system generates and enhances only individual play-ing. In fact, it was also possible to observe that when the child plays with a friend, the use of the system also improves their flow state. These results highlight the pedagogical framework of reflexive interaction, which proves to have many links with the socio-constructivist approach (Bruner, 1983; Vygotsky, 1962).

As far as exposure to the system is concerned, the experiment shows that reflexive interaction keeps the level of flow experience high and constant over time, thereby strengthening the creative experience and the child's learning. This con-dition could have an important effect on children's learning processes, because it broadens the attention span and increases the intrinsic motivation.

In the field of child-machine interaction and technologyenhanced learning, these results could have a significant impact because they support the hypothesis that reflexive

Table 4. Percentage of each behaviour, in total and in each task.

\begin{tabular}{|c|c|c|c|c|c|}
\hline & Total & $\mathrm{T} 1$ & $\mathrm{~T} 2$ & $\mathrm{~T} 3$ & $\mathrm{~T} 4$ \\
\hline Focused attention & 75.1 & 82 & 85.1 & 66.4 & 71.4 \\
\hline Clear-cut feedback & 59.8 & 60.9 & 67.6 & 55.3 & 57.9 \\
\hline Control of situation & 85.4 & 85.6 & 86.4 & 83.9 & 85.9 \\
\hline Pleasure & 80.7 & 69.4 & 82.1 & 82.5 & 87 \\
\hline
\end{tabular}




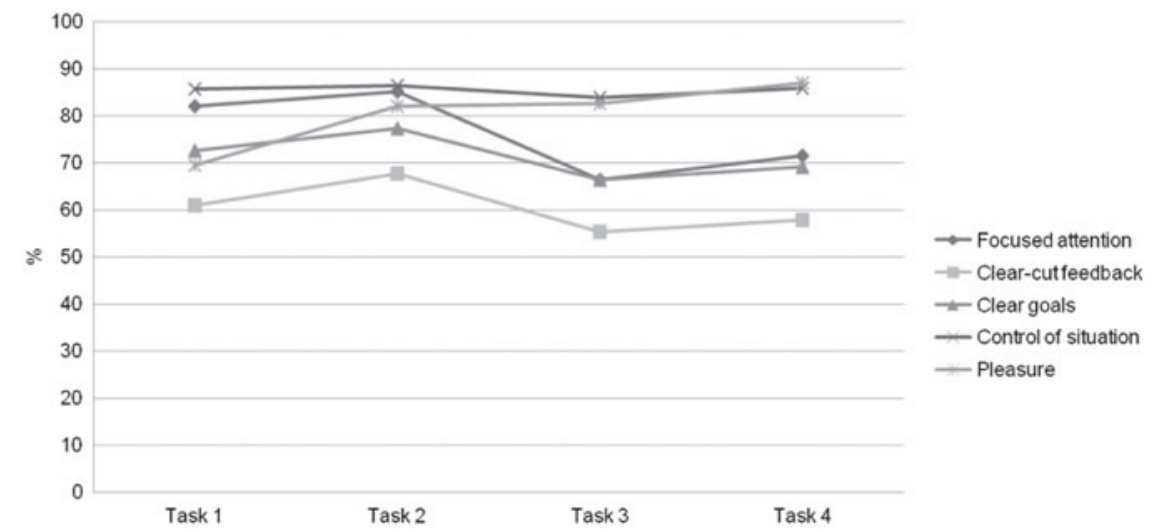

Fig. 9. The behaviours: Trend of focused attention, clear-cut feedback, clear goals, control of situation and pleasure, in all sessions and all subjects.

interaction is able to create a flow environment during childmachine interaction. They also demonstrate, as suggested by Finneran and Zhang (2005) and Leman et al. (2010), that flow is an efficient tool to investigate child-machine interaction analytically.

An interesting aspect highlighted by our study is that the MIROR-Impro tends to generate a higher level of flow in older children and several improvements need to be discussed to better adapt the system to younger children. These data, however, could also give rise to two considerations: the first is that the flow of younger children could be different and be expressed differently than in older children. The second is that the proposed flow grid might be more suitable for observing the flow of older children. The grid could be adjusted to younger children according to the flow indicators proposed by Custodero $(2005,2011)$.

Certain results concerning the set-up have also highlighted some issues that could be improved. In particular, it could be useful to revise the reflexive quality of the reply of the MIROR-Impro. For example, in the MIROR-Impro 'the system starts the output with the input starting note, and produces a continuation from there (and) the system ends the melody with the input ending note' (User Manual MIRORImpro v.2.5, p. 16). However, is the child able to memorize and recognize the first and the last note that $\mathrm{s} / \mathrm{he}$ just played, considering that the child played a long phrase? In our opinion, $\mathrm{s} / \mathrm{he}$ is not, for at least two reasons: the main reason is that just one note may not be sufficient to create a significant perceptual cue to be memorized and recognized after such a long time, not only in young children but also in adults (e.g. Deliège, 2001). This implies that memorizing the first and last note played is a very difficult task for the children. This does not mean that the results do not confirm the hypothesis that MIROR-Impro generates flow state, but rather that further adjustments need to be introduced to the set-up to improve the ability of the MIROR-Impro to act as a flow machine. For example, allowing the user to set the degree of repetition and variation on the basis of the user age and expertise. In conclusion, the reflexive qualities of the set-up of the MIROR-Impro could be enhanced by taking into account the auditory perception of similarity (e.g. Deliège, 2001; Toiviainen, 2007).

Further considerations derive from the experimental setting and the interfaces used by children, namely the keyboard and the monitor of the computer. In fact, while in the pilot protocol (Addessi et al., 2006) the only interface between the children and the system was the keyboard, in the experiment that we present in this paper, the children could also watch the graphic interface of the MIROR-Impro on the computer monitor. In the pilot protocol, then the children interacted with the system exclusively through the sound, while in the present protocol they also had visual interaction. We noted that the children were very attracted by the monitor interface and that sometimes they were even distracted by it and their playing became fragmentary and discontinuous. We might argue that the presence of the monitor interface was one of the causes of the lower percentage of flow in the second experiment. Above all, we believe that an interaction exclusively based on the sound generates, in this particular reflexive environment, a higher level of flow. This observation, if verified by further experiments, could be very significant for the consequences it could have in the field of child-machine interaction and technology-enhanced learning, as far as the implementation of an interface is concerned. This aspect is also fundamental in the field of music education, as it highlights the importance to develop systems that are able to establish a meaningful interaction with children based on the sound and therefore able to develop in the children the attitude to 'thinking in sound', which is one of the principal aims of music education (McPherson, 2005).

As far as the flow grid is concerned, it generally worked well, although several adjustments could be made in the future to make it more adaptive to young children and to capture the dynamic evolution of the flow experience. A comparison with the grid we used in the previous experiment highlights that several improvements have already been introduced in the new flow grid developed with the Observer software. 
In particular, in the pilot protocol each observer could only assess the value of each behaviour for the full task, while with the flow grid developed with the Observer software, the observer could register the presence, the frequency, the duration and the value of each variable, second by second, in each task. Furthermore, we implemented the data profile based on nesting of the behaviours, obtained by selecting only the high levels (modifier $=3$ ) of each behaviour, which allows the Observer to generate the presence of the flow automatically. Therefore, the more analytical grid made it possible to check and measure each variable in more detail and allowed various comparisons and data analyses to be made. However, some limitations should be considered, in particular the fact that only five variables were registered, while in Csikszentmihalyi (1996) there are nine. In fact, we registered only the variables that were feasible in terms of observable behaviours, such as focused attention, clear-cut feedback, control of situation, clear aims and pleasure.

\section{Conclusion}

This paper presented a study aiming to measure the flow experience of children playing with the MIROR-Impro, a flow machine for childhood music education, implemented in the framework of the MIROR Project. The study also aimed to create and test a grid to analyse and measure the flow state of children interacting with a flow machine.

The results obtained with the flow grid allowed us to confirm the hypothesis that the use of the system and reflexive interaction can enhance flow experience and highlighted several areas where the prototype could be improved. By means of the flow grid it was possible to observe and measure the optimal experience and the emotional states as described by Csikszentmihalyi (1996) in an analytic and efficient way in 4 and 8-year-old children playing with the MIROR-Impro. As well as supporting the hypothesis of the flow machine (Pachet, 2004), the grid could also prove useful for other experiments, in particular for measuring the state of creativity in child-machine interaction, an aspect that is very important in the field of technology-enhanced learning and human-machine interaction (Leman et al., 2010) and more in general in music education (Custodero, 2005; McDonald et al., 2006). Further-more, it may also be applied for user experience testing of other components of the MIROR platform, in particular that of the MIROR-Body Gesture. In this case, it would be helpful to integrate the flow grid with other dimensions and tools (e.g. Jackson \& Eklund, 2004; Leman et al., 2010; Lesaffre et al., 2009; Nijs et al., 2012). From a pedagogical point of view, the grid could be a useful tool for music teachers as well as general teachers to observe the state of flow and creativity in children during musical activities. The flow grid could be used for both offline (recorded videos) and online observations.

In the future, we are planning to adopt the flow grid for several different aims. Firstly, the flow grid could be used by the MIROR platform, to carry out the observations in an automated way through a system of motion capture. This way, the MIROR platform will be further enriched with a feedback tool for the user (the child but also the teacher). This tool will allow the user to have feedback on his/her experienced state of flow, either in real-time or deferred. The results could also suggest how to create different variations of the system, driven by the observation of child-machine interaction in order to better understand what induces experiences of flow and feelings of presence (Riva et al., 2004) in young children. We plan to adopt the flow grid for an in-depth study of the correlations between flow experience and children's musical improvisation, children's listening conducts, and the role of the teacher. In order to analyse in more detail whether the reflexive behaviour of the system affects the children's flow state, further data analyses are currently being performed by means of an auditive analysis, whereby different levels of repetition and variation in the system's reply will be grasped and then correlated with the flow results. Finally, the grid may be used for future experimental studies on human interaction with flow machines and, more generally, in the field of studies on child-machine interaction.

\section{Acknowledgements}

This article is a revised and extended version of the paper published in E. Cambouropoulos, C. Tsougras, P. Mavromatis, \& K. Pastiadis (Eds.) (2012), Proceedings of the 12th International Conference on Music Perception and Cognition (ICMPC) and the 8th Triennial Conference of the European Society for the Cognitive Sciences of Music (ESCOM) (pp. 20-30), Thessaloniki, Greece. Adapted with permission.

Funding:This work was partially supported by the EU-ICT Project MIROR (Musical Interaction Relying On Reflexion, www.mirorproject.eu).

\section{References}

Addessi, A.R. (2014). Developing a theoretical foundation

for the reflexive interaction paradigm with implications for training music skill and creativity. Psychomusicology: Music, Mind, and Brain, 24(3), 214-230.

Addessi, A.R. (2013). Child/machine interaction in reflexive environment. The MIROR Platform. In R. Bresin (Ed.), Proceedings of the Sound and Music Computing Conference (SMC2013) (pp. 95-102). Berlin: Logos Verlag Berlin GmbH.

Addessi, A.R., Ferrari, L., Carlotti, S., \& Pachet, F. (2006). Young children musical experience with a flow machine. In M. Baroni, A.R. Addessi, R. Caterina, \& M. Costa (Eds.), Proceedings of the 9th International Conference on Music Perception and Cognition (ICMPC) and 6th Triennial Conference of the European Society for the Cognitive Sciences of Music (ESCOM) (pp. 1658-1665). Bologna: Bononia University Press. 
Addessi, A.R., \& Pachet, F. (2005). Experiments with a musical machine. Musical style replication in 3/5-year-old children. British Journal of Music Education, 22(1), 21-46.

Addessi, A.R., \& Pachet, F. (2006). Young children confronting the Continuator, an interactive reflective musical system Musicae Scientiae, 10( Suppl. 1), 13-39.

Addessi, A.R. \& Volpe, G. (2011). The MIROR Project. Towards ubiquitous learning. Proceedings of the Sixth European Conference on Technology Enhanced Learning (pp. 15-28). Berlin: Springer Verlag.

Assayag, G., \& Dubnov, S. (2004). Using factor oracles for machine improvisation. Soft Computing - A Fusion of Foundations, Methodologies and Applications, 8(9), 604-610.

Assayag, G., Dubnov, S. \& Delerue, O. (1999). Guessing the composer's mind: Applying universal prediction to musical style. In Proceedings of International Computing Music Conference Beijing, China (pp. 496-499). Ann Arbor, MI: Michigan Publishing.

Anzieu, D. (1996). Les enveloppes psychiques. Paris: Dunod.

Bruner, J. (1983). Child's talk: Learning to use language. New York: Norton.

Burnard, P. (2006). Understanding children's meaning-making as composers. In I. Deliège, \& G.A. Wiggins. (Eds.), Musical creativity. Multidisciplinary research in theory and practice (pp. 111-132). Hove: Psychology Press.

Byrne, C., McDonalds, R., \& Carlton, L. (2003). Assessing creativity in musical composition: Flow as an assessment tool. British Journal of Music Education, 20(3), 277-290.

Chartrand, T.L., \& Bargh, J.A. (1999). The chameleon effect: The perception-behaviour link and social interaction. Journal of Personality and Social Psychology, 76, 893-910.

Cope, D. (1996). Experiments in musical intelligence. Madison, WI: A-R Editions.

Csikszentmihalyi, M. (1990). Flow: The psychology of optimal experience. New York: Harper \& Row.

Csikszentmihalyi, M. (1996). Creativity: Flow and the psychology of discovery and invention. New York: HarperCollins.

Csikszentmihalyi, M. (1997). Finding flow: The psychology of engagement with everyday life. New York: Basic Book.

Csikszentmihalyi, M., \& Csikszentmihalyi, I.S. (Eds.) (1988). Optimal experience. Psychological studies of flow in consciousness. New York: Cambridge University Press.

Custodero, L.A. (2005). Observable indicators of flow experience: Adevelopment perspective on musical engagement in young children from infancy to school age. Music Education Research, 7(2), 185-209.

Custodero, L.A. (2011). The call to create: Flow experience in music learning and teaching. In D. Hargreaves, D. Miell, \& R. MacDonald. (Eds.), Musical imagination. Multidisciplinary perspectives on creativity, performance and perception (pp. 369-384). Oxford: Oxford University Press.

Delalande, F. (1993). Le condotte musicali. Bologna: CLUEB.

Deliège, I. (2001). Similarity perception - Categorization - Cue abstraction. Music Perception, 18, 233-243.

Dissanayake, E. (2000). Antecedents of the temporal arts in early mother-infant interaction. In N.L. Wallin, \& B. Merker
(Eds.), The origin of music (pp. 389-410). Cambridge, MA: Massachusetts Institute of Technology Press.

Ferrari, L., \& Addessi, A.R. (2014). A new way to play music together: The Continuator in a classroom setting. International Journal of Music Education, 32(2), 171-184.

Finneran, C.M., \& Zhang, P. (2005). Flow in computer-mediated environments: Promises and challenges. Communications of the Association for Information Systems, 15, 82-101.

Fogel, A. (2000). Oltre gli individui: un approccio storicorelazionale alla teoria e alla ricerca sulla comunicazione. In M.L. Genta (Ed.), Il rapporto madre-bambino (pp. 123-161). Rome: Carocci.

Fober, D., Letz, S., \& Orlarey, Y. (2007). VEMUS - Feedback and groupware technologies for music instrument learning. In C. Spyridis, A. Georgaki, G. Kouroupetroglou, \& C. Anagnostopoulou (Eds.), Proceedings of the 4th Sound and Music Computing Conference (SMC07), Lefkada, Greece, 117-123.

Gratier, M., \& Apter-Danon, G. (2008). The musicality of belonging: Repetition and variation in mother-infant interaction. In C. Trevarthen, \& S. Malloch (Eds.), Communicative musicality (pp. 301-327). Oxford: Oxford University Press.

Gonzalez Thomas, N., Pasquier, Ph., Eigenfeldt, A. \& Maxwell, J.B. (2013). A methodology for the comparison of melodic generation models using META-MELO. In A. De Souza Britto Jr, F. Gouyon, \& S. Dixon. (Eds.), Proceedings of 14th International Society for Music Information Retrieval Conference (ISMIR2013) (pp. 561-566). Curitiba, Brazil: ISMIR.

Imberty, M. (2005). La musique creuse le temps. De Wagner à Boulez. Musique, psychologie, psychoanalyse. Paris: L'Harmattan.

Jackson, S., \& Eklund, R. (2004). The flow scale manual. Morgantown: Fitness Information Technology.

Kurtág, G., Di Santo, J.L., Desainte-Catherine, M. \& Guillem, $\mathrm{Ph}$. (2007). Pédagogie de l'électroacoustique: du geste musical à la composition assistée par l'ordinateur. In Proceedings of the 12èmes Journées d'Informatique Musicale (pp. 73-78). Lyon: GRAME.

Lakin, J.L., \& Chartrand, T.L. (2003). Using nonconscious behavioral mimicry to create affiliation and rapport. Psychological Science, 14, 334-339.

Leman, M., Lesaffre, M., Nijs, L., \& Deweppe, A. (2010). User-oriented studies in embodied music cognition research. Musicae Scientiae, 14 (Suppl. 2), 203-224.

Lesaffre, M., Nijs, L., \& Leman, M. (2009). Interacting with music mediation technology for hearing impaired - first tests with normal hearing subjects. In J. Louhivuori, T. Eerola, T. Himberg, S. Saarikallio, \& P.-S. Eerola (Eds.), Proceedings of the 7th Triennial Conference of the European Society for the Cognitive Sciences of Music (ESCOM2009) (pp. 267-270). Jyvaskyla, Finland.

Malloch, S. (2000). Mothers and infants and communicative musicality. Musicae Scientiae, 3(Suppl. 1), 29-58.

Maxwell, J.B., Eigenfeldt, A., Pasquier, P. \& Gonzalez Thomas, N. (2012). MusiCOG: A cognitive architecture for music learning and generation. In Proceedings of the 9th Sound 
and Music Computing conference (SMC2012) (pp. 521-528). Copenhagen, Denmark.

McDonald, R., Byrne, C., \& Carlton, L. (2006). Creativity and flow in musical composition: An empirical investigation. Psychology of Music, 34(3), 292-306.

McPherson, G. (2005). From child to musician: Skill development during the beginning stage of learning an instrument. Psychology of Music, 33, 5-35.

Meeùs, N. (1993). Le rapports associatifs comme déterminants du style. Analyse Musicale, 32, 9-13.

Nadel, J., \& Butterworh, G. (Eds.) (1999). Imitation in infancy. Cambridge: Cambridge University Press.

Nattiez, J.J. (1987). Musicologie générale et sémiologie. Paris: Bourgois.

Nijs, L., Moens, B., Lesaffre, M., \& Leman, M. (2012). The Music Paint Machine: Stimulating self-monitoring through the generation of creative visual output using a technologyenhanced learning tool. Journal of New Music Research, 41(1), 79-101.

O’Neill, S.A., \& McPherson, G. (2002). Motivation. In R. Parncutt, \& G. McPherson (Eds.), The science and psychology of music (pp. 31-47). Oxford: Oxford University Press.

Pachet, F. (2003). Music interaction with style. Journal of New Music Research, 32(3), 333-341.

Pachet, F. (2004). On the design of Flow Machine. In M. Tokoro (Ed.), A learning zone of one's own: Sharing representations and flow in collaborative learning environments (pp. 111-134). The Netherlands: IOS Press.

Pachet, F. (2006). Creativity studies and musical interaction. In I. Deliège, \& G.A. Wiggins. (Eds.), Musical creativity. Multidisciplinary research in theory and practice (pp. 347358). Hove: Psychology Press.

Pachet, F., \& Addessi, A.R. (2004). Children reflect on their own playing style: Experiments with Continuator and children. ACM Computers in Entertainment, 1(2), 1-13.

Pachet, F., Roy, P. \& Barbieri, G. (2011). Finite-Length Markov processes with constraints. In Proceedings of Twenty-Second International Joint Conference on Artificial Intelligence (IJCAI2011) (pp. 635-642). Menlo Park, CA: AAAI/International Joint Conference on Artificial Intelligence.
Papousěk, M. (1995). Le comportement parental intuitif, source cachée de la stimulation musicale dans la petite enfance. In I. Deliège, \& J. Sloboda (Eds.), Naissance et développement du sens musical (pp. 101-130). Paris: Presses Universitaires de France.

Riva, G., Waterworth, J., \& Waterworth, E. (2004). The layers of presence: A bio-cultural approach to understanding presence in natural and mediated environments. Cyber Psychology \& Behavior, 7(4), 402-415.

Rizzolatti, G., Fadiga, L., Gallese, V., \& Fogassi, L. (1998). Premotor cortex and the recognition of motor actions. Cognition Brain Research, 3(2), 131-141.

Ruwet, N. (1966). Méthodes d'analyse en musicologie. Revue belge de Musicologie, 20, 65-90.

Sheridan, M., \& Byrne, C. (2002). Ebb and flow of assessment in music. British Journal of Music Education, 19(2), 135-143.

Smolej Fritz, B., \& Avsec, A. (2007). The experience of flow and subjective well-being of music students. Horizons of Psychology, 16(2), 5-17.

Stern, D. (2004). The present moment in psychotherapy and in everyday life. New York: Norton.

Teddlie, C., \& Tashakkori, A. (2009). Foundations of mixed methods research. Integrating quantitative and qualitative approaches in the social and behavioural sciences. Thousand Oaks, CA: SAGE Publications.

Toiviainen, P. (Ed.) (2007). Similarity perception in listening to music [Special Issue]. Musicae Scientiae, 11.

Trevarthen, C. (2000). Musicality and the intrinsic motive pulse: Evidence from human psychobiology and infant communication. Musicae Scientiae, 3(Suppl. 1), 155-215.

Triviño-Rodriguez, J.L., \& Morales-Bueno, R. (2001). Using multiattribute prediction suffix graphs to predict and generate music. Computer Music Journal, 25(3), 62-79.

Volpe, G., Varni, G., Addessi, A.R. \& Mazzarino, B. (2012). BeSound: Embodied reflexion in childhood. In Proceedings of the 11th International Conference on Interaction Design and Children (IDC2012) (pp. 172-175). New York, ACM.

Vygotsky, L. (1962). Thought and language. Cambridge, MA: Massachusetts Institute of Technology Press. 